\title{
Carbon Nitride as a Heterogeneous Visible-Light Photocatalyst for the Minisci Reaction and Coupling to $\mathrm{H}_{2}$ Production
}

Received 00th January 20xx, Accepted 00th January 20xx

DOI: $10.1039 / x 0 x \times 00000 x$

\author{
Arjun Vijeta and Erwin Reisner*
}

Cyanamide functionalised Carbon nitride powder is reported as a photocatalyst for direct Minisci-type coupling of heteroarenes with ethers, alcohols, and amides using atmospheric oxygen as the oxidant at room temperature. This mild protocol displays broad substrate scope, good functional group tolerance and the catalyst can be easily isolated and reused for several cycles. It thereby addresses the two major limitations of previously reported photoredox-mediated Minisci reactions: (i) use of expensive and potentially harmful non-recyclable photocatalysts, and (ii) requirement of a stoichiometric amount of strong chemical oxidant. Finally, using platinum as a co-catalyst with the carbon nitride allows this light-mediated reaction to generate two valueadded products under an anaerobic atmosphere - functionalised heteroarenes and $\mathrm{H}_{2}$ fuel.

Harnessing visible light to store energy in the form of chemical bonds has attracted wide attention in the field of artificial photosynthesis for decades. Over the years, this approach has been extensively used for overall water splitting to produce $\mathrm{H}_{2}$ and $\mathrm{CO}_{2}$ utilisation. ${ }^{1,2}$ The potential to use visible light to drive catalytic reactions in organic synthesis, however, has not been fully realised until 2008. ${ }^{3-5}$ Since then, light-mediated catalysis, also known as photoredox catalysis, has enabled the discovery of a wide array of novel synthetic methodologies. ${ }^{6}$ Homogeneous photocatalysts such as metal-based polypyridyl complexes of ruthenium and iridium or organic dyes, especially eosin $\mathrm{Y}$ and acridinium salts, have been the major contributor in photoredox catalysis. 7,8 While recent advances have provided versatility in these photocatalysts, there appears plenty of scope for inexpensive, non-toxic, stable and scalable alternatives.

A promising photocatalyst material is polyheptazine-based graphitic carbon nitride $\left(\mathrm{CN}_{\mathrm{x}}\right.$ or $\left.g-\mathrm{C}_{3} \mathrm{~N}_{4}\right) .{ }^{9}$ This low-cost solid material $\left(<0.1 \$ \mathrm{~g}^{-1}\right.$ on lab-scale) has a band gap of

Department of Chemistry, University of Cambridge, Lensfield Road, Cambridge CB2 1EW, U.K.

Email: reisner@ch.cam.ac.uk

Electronic Supplementary Information (ESI) available: [details of any supplementary information available should be included here]. See DOI: 10.1039/x0xx00000x approximately $2.7 \mathrm{eV}$ with adjustable band positions that can be tuned by structural or chemical modification. ${ }^{10}$ Moreover, its robust structure provides photo and chemical stability with the advantages of heterogeneous catalysis: ease of separation and reusability. In the last decade, carbon nitride has been used as photocatalyst in various chemical transformations, including selective oxidation of alcohols to aldehydes or ketones, amines to imines and sulfides to sulfoxides. ${ }^{11-13}$ Recently, our group has reported a closed atom-economical photocatalytic system for selective oxidation of alcohols to aldehyde coupled with the reduction of aqueous protons to produce $\mathrm{H}_{2} \cdot{ }^{14,15}$ This work employed cyanamide functionalised carbon nitride as a photocatalyst, which shows better activity than intrinsic carbon nitride due to improved photogenerated charge separation. ${ }^{16-}$ 18 This property motivated us to further explore complex organic transformations using carbon-based photocatalysts.

Heteroaromatic moieties are present in various natural products, pharmaceuticals, and ligand scaffolds. ${ }^{19}$ An efficient and sustainable approach for the direct functionalisation of $\mathrm{C}$ $\mathrm{H}$ bonds in heteroarenes has therefore become a very important goal in organic synthesis. The Minisci reaction is a known strategy for such functionalisation, involving the radical coupling of electron-deficient heteroarenes with oxidativelygenerated nucleophilic radicals. ${ }^{20,21}$ Classically, this reaction requires an activated moiety as a radical source, and metal salts or stoichiometric amount of peroxides at an elevated temperature. ${ }^{21,22}$

Over the years, various studies have been performed to improve the traditional Minisci conditions including work on light-mediated catalysis, which elegantly circumvented the necessity of elevated temperature. ${ }^{23-26}$ Photocatalyzed Minisci coupling has recently been developed for the alkylation of $\mathrm{N}$ heteroarenes, $\alpha$-heteroarylation of ethers and $N$-protected secondary amines, but the chemistry has not yet been explored for the heteroarylation of alcohol and amides. ${ }^{27-32}$ Nevertheless, these Minisci protocols employ expensive and potentially toxic Ir and Ru metal complexes as photocatalysts and still require activated moieties as the nucleophilic radical source, and a stoichiometric amount of strong chemical 
oxidants.

In light of these limitations, we report here a visible lightmediated Minisci coupling of $\mathrm{N}$-heteroarenes with ethers, alcohols and amides in the presence of aerobic oxygen as the oxidant, using a cyanamide functionalised carbon nitride photocatalyst (Scheme S1). Furthermore, we demonstrate that the Minisci reaction can be coupled to the co-production of molecular hydrogen $\left(\mathrm{H}_{2}\right)$ under anaerobic conditions, demonstrating the ability to couple complex organic synthesis with fuel production. ${ }^{33}$

Table 1. Standard condition for Minisci coupling

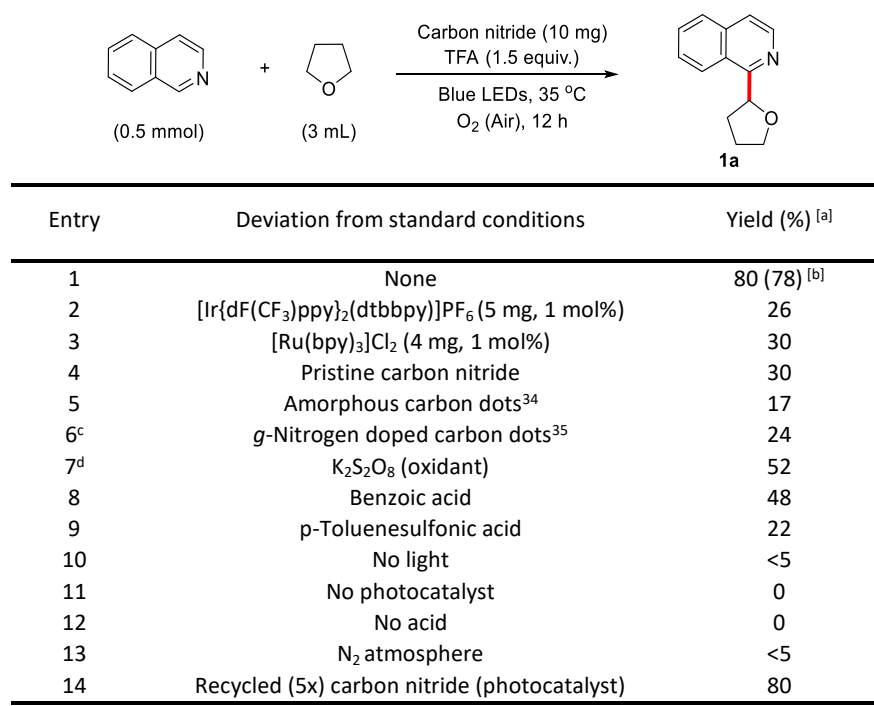

[a] Yield determined by ${ }^{1} \mathrm{H}$ NMR using 1,3,5-trimethoxybenzene $(0.1 \mathrm{mmol})$ as the internal standard. [b] Isolated yield. [c] $3 \mathrm{mg}$ of graphitic nitrogen doped carbon dots. [d] $1 \mathrm{~mL}$ of water as solvent under inert atmosphere.

Carbon nitride was synthesised by calcination of melamine at $550{ }^{\circ} \mathrm{C}$, followed by post-synthetic cyanamide functionalisation using potassium thiocyanate (Figure S1). ${ }^{16}$ We envisioned that upon blue-light irradiation the strongly oxidising valence band (VB) of this polymeric material (potential of $\mathrm{VB}\left(\mathrm{E}_{\mathrm{VB}}\right)=+2.2 \mathrm{~V}$; conduction band $\left(E_{C B}\right)=-0.5 \vee$ vs standard hydrogen electrode, $\mathrm{SHE}$ ) would allow the direct oxidation of ethers, alcohols and amides to generate the corresponding open-shell nucleophilic radical species (for THF, DMF, and $\mathrm{MeOH} \mathrm{E}_{1 / 2}$ ox $>+1.84 \mathrm{~V}$ vs $\mathrm{SHE}) .{ }^{17,36}$ Subsequently, the radical species can be engaged in Minisci-type coupling with $\mathrm{N}$-heteroarenes.

We started examining the Minisci-type coupling of isoquinoline ( $0.5 \mathrm{mmol}, 1.0$ equiv.) with THF ( $3 \mathrm{~mL}$ ) as substrate and solvent under various conditions (Table 1; see Figures S2-S5 for experimental set-up and optimisation of conditions). An excellent yield of $80 \%$ for the desired coupling product 1a was obtained in the presence of carbon nitride $(10 \mathrm{mg})$ as a photocatalyst, trifluoroacetic acid (TFA; 1.5 equiv.) as the proton source and aerobic oxygen as the oxidant under irradiation using $14.4 \mathrm{~W}$ blue LEDs with $\lambda_{\max }=470 \mathrm{~nm}$ (entry 1 ). The activity of carbon nitride was subsequently compared to other photocatalysts. Commonly used precious metal photocatalysts were found to produce a lower yield (entries 23). Pristine carbon nitride showed a diminished yield (entry 4), which can be explained by the lack of cyanamide-functionality to improve the substrate/carbon nitride interaction as well as charge separated state lifetime. ${ }^{14,16}$ Recently, homogenous carbon dots have emerged as a low-cost, stable and non-toxic light harvester in photocatalytic fuel synthesis systems. ${ }^{34,35,37}$ Albeit at lower yields than pristine carbon nitride, carbon dots were also identified to be effective for the coupling (entries 5 and 6), which motivates their future exploration in photocatalysed organic transformations. Using other oxidants and acids resulted in a diminished yield (entries 7-9). Control experiments show the necessity of light, photocatalyst, acid, and oxidant for the reaction (entries 10-13).

Table 2. Heteroaromatic substrate scope
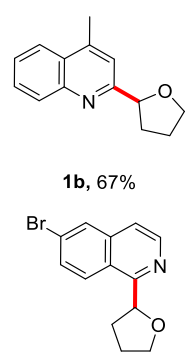

1f, $52 \%$

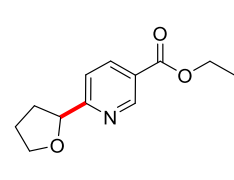

$1 \mathrm{j}, 60 \%$

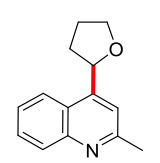

1 c, $83 \%$<smiles>[BH3-]</smiles>

$\mathrm{Br}$

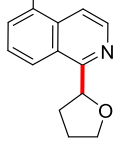

$1 \mathrm{~g}, 50 \%$

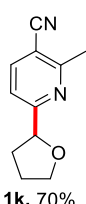

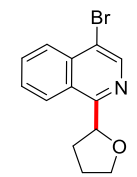

1d, $58 \%$

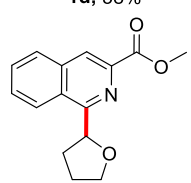

$1 \mathrm{~h}, 60 \%$

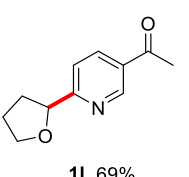

${ }^{a}$ Isolated yields are reported. Reaction time varied from 4-48 h (see SI).

Following the establishment of the optimised standard conditions, substrate scope of the photocatalytic Minisci reaction was investigated. First, we focused on examining the scope of $\mathrm{N}$-heteroarenes (Table 2). Various electron-deficient heteroarenes including quinolines (1a-c), isoquinolines (1d-h) and pyridines (1i-I) were observed to readily couple with THF at the most electrophilic position in good to excellent yield. 4methyl and 2-methyl quinoline resulted in selective coupling with THF at the $\mathrm{C} 2$ and $\mathrm{C} 4$ positions, respectively (1b-c, 67-83\% yield). Moreover, sensitive functional groups such as bromo substitution at $\mathrm{C} 4, \mathrm{C} 5$, and $\mathrm{C} 6$ positions and ester at the $\mathrm{C} 3$ position of isoquinoline were well tolerated, along with 3methyl isoquinoline (1d-h, $50-66 \%$ yield). Notably, pyridines with electron-withdrawing groups were found to be amenable to the protocol (1i-I, $60-75 \%$ yields).

Next, we investigated the scope of ethers (Table 3). The cyclic ethers coupled efficiently with isoquinolines, but no reactivity was observed for acyclic ethers (e.g., dimethoxy ethane and tert-butyl ether), which can be attributed to their higher oxidation potential. ${ }^{38}$ Tetrahydropyran, 1,4-dioxane and 1,3dioxalane were studied and found to be amenable to the coupling with a good yield (2a-c, yield 50-83\%). The 1,3dioxalane contains two different $\alpha$-oxy positions $\mathrm{C}-\mathrm{H}$ bonds, resulting in a mixture of coupling products ( $\mathrm{C} 2$ : $\mathrm{C} 4$ coupling ratio $=3.4: 1)$.

Furthermore, the Minisci coupling was applied to primary alcohols (Table 3). Generally, alcohols are employed as an alkyl 
source with the subsequent loss of the hydroxyl groups in the light-mediated reactions. ${ }^{29,39-41}$ We observed direct functionalisation of the $\alpha-\mathrm{sp}^{3} \mathrm{C}-\mathrm{H}$ bond of primary alcohols with isoquinoline. Various primary alcohols including methanol, ethanol, n-propanol, and n-butanol afforded $\alpha$-arylated alcohols in moderate to good yield (3a-d, $25-53 \%$ yields). The diversity of the process was expanded to amides and good to excellent yields were obtained. Isoquinoline, 4bromoisoquinoline and lepidine selectively couple with $N, N$ dimethylformamide (DMF) and $\mathrm{N}, \mathrm{N}$-dimethylacetamide (DMA) (4a-f, 71-96\% yield). Moreover, an expected mixture of coupling products was observed for $\mathrm{N}$-methyl-2-pyrrolidone due to the presence of two different $\alpha$-amide $\mathrm{C}-\mathrm{H}$ bonds as a potential nucleophilic radical source $(\mathbf{4 g}, 97 \%$ yield).

Table 3. Scope of ethers, alcohols and amides ${ }^{[a]}$

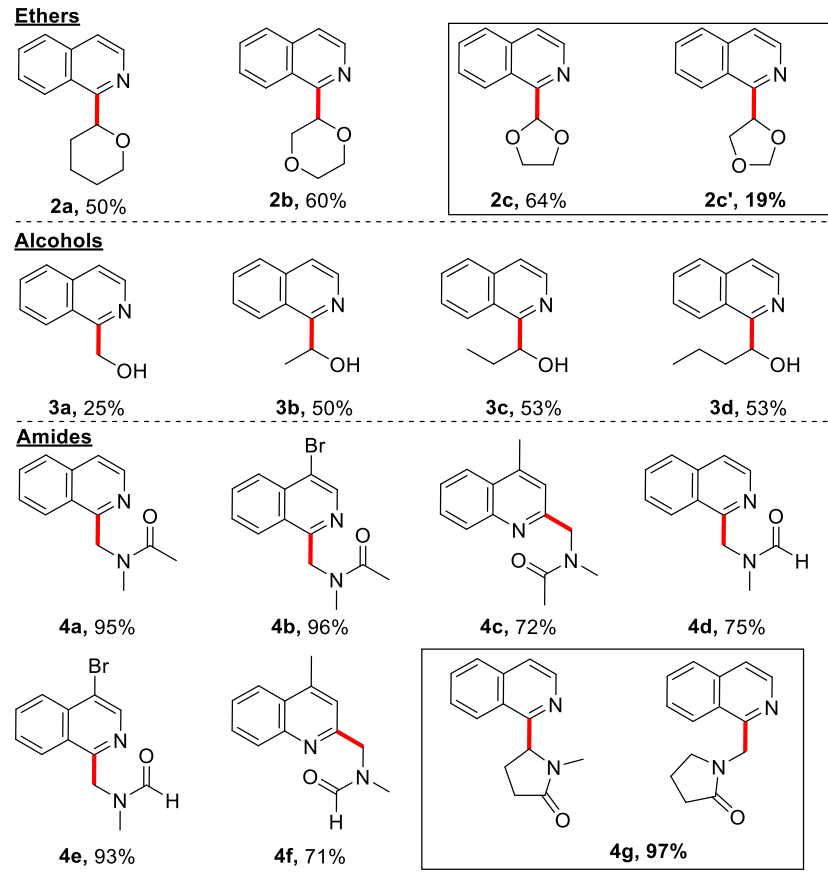

a Isolated yields are reported. Reaction time varied from $11-48 \mathrm{~h}$ (see $\mathrm{SI}$ ).

After exploring the scope of the reaction, we studied the reusability of the carbon nitride photocatalyst. The used carbon nitride was washed two times with water followed by acetone and re-employed in another photocatalytic reaction (Figure S6). Notably, activity remained the same for five continuous experiments (Table 1, entry 14), even though partial loss of cyanamide functionality in carbon nitride was observed (Figure S7). The carbon nitride can be fully recovered by centrifugation and basic wash retaining the yellow colour. The recyclability demonstrates the advantage of carbon nitride as a heterogeneous photocatalyst.

We then investigated the mechanism of the reaction. The employed carbon nitride is known to change colour from yellow to blue upon irradiation in the presence of electron donors under oxidant free conditions by forming long-lived trapped electrons with a lifetime of over 10 hours. ${ }^{14,17}$ Consistent with this behaviour, we observed a dark blue colouration of the reaction suspension along with only traces of product when the reaction was performed in the absence of $\mathrm{O}_{2}$ (Figure 1). This supports that THF is acting as an electron donor for carbon nitride. No colour change was observed during irradiation in the presence of $\mathrm{O}_{2}$ as the photogenerated electrons in carbon nitride are readily quenched. Furthermore, replacing $\mathrm{O}_{2}$ with $\mathrm{H}_{2} \mathrm{O}_{2}$ under inert conditions yielded $40 \%$ of desired product (conversion 64\%), suggesting that other oxygen reactive species can also act as electron acceptor in the reaction. The in-situ generation of $\mathrm{H}_{2} \mathrm{O}_{2}$ from $\mathrm{O}_{2}$ was confirmed by an iodometry essay (Figure $\mathrm{S} 8$ ).

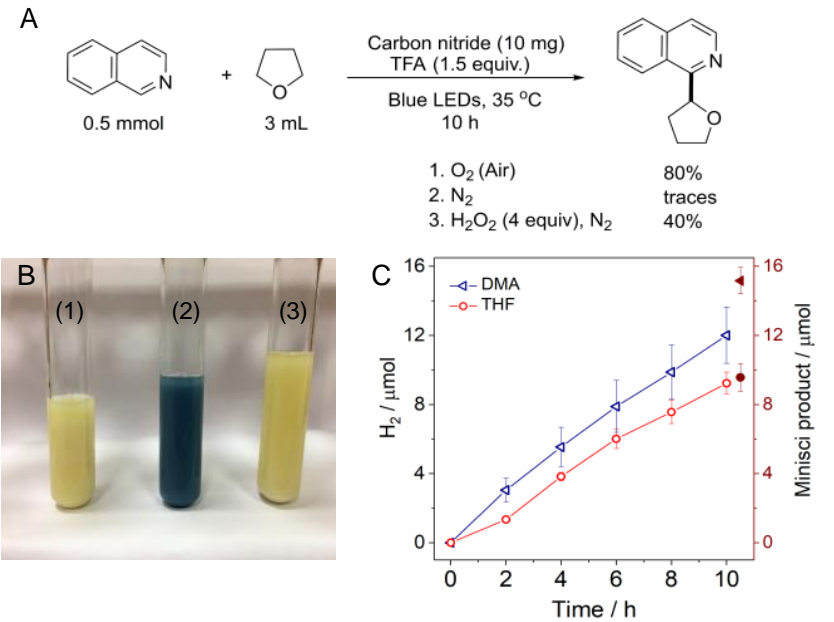

Figure 1. Mechanistic studies: (A) Reaction under different atmospheres. (B) Colour of above reaction mixtures after LED irradiation in the presence of (1) $\mathrm{O}_{2},(2) \mathrm{N}_{2}$, and, (3) $\mathrm{H}_{2} \mathrm{O}_{2}$ in $\mathrm{N}_{2}$. (C) Photocatalytic $\mathrm{H}_{2}$ production; reaction condition: Isoquinoline $(50 \mu \mathrm{mol})$, THF or DMA $(0.3 \mathrm{~mL}$ ), TFA (1.5 equiv.), carbon nitride $(5 \mathrm{mg})$, nanoparticles of Pt (1000 ppm in water, $15 \mu \mathrm{L}$ ) and an aqueous phosphate solution $(0.1 \mathrm{M}, 2.7 \mathrm{~mL}$ ) irradiated with blue LEDs. Product yields were obtained by gas chromatography for $\mathrm{H}_{2}$ (hollow symbols) and by ${ }^{1} \mathrm{H}$ NMR spectroscopy for the Minisci oxidation product (filled symbols). See $\mathrm{SI}$

Furthermore, Pt nanoparticles were employed as a $\mathrm{H}_{2}$ evolving catalyst to sustainably quench the photogenerated electrons in the absence of $\mathrm{O}_{2}$ (Figure 1C). Remarkably, we observed the desired Minisci coupling product along with $\mathrm{H}_{2}$ formation. This indicates that the electrons released from the oxidative coupling reaction are quenched by the $\mathrm{VB}$ of carbon nitride and, subsequently, the $\mathrm{CB}$ electrons in carbon nitride can be transferred to the Pt catalyst for proton reduction. Moreover, this suggests that our Minisci reaction can be sustainably used for generation of two value-added products, solar chemical and solar fuel.

A tentative mechanism for the photocatalytic Minisci reaction is proposed in Scheme 1 based on the observations and literature reports. Visible light irradiation of the photocatalyst carbon nitride produces a photoexcited state that acts as a strong oxidant. The VB engages in a single electron oxidation with $\mathbf{5}$ (ethers, alcohols and amides) to afford the nucleophilic radical species $6 .{ }^{18,36}$ The oxidised species 6 then couples to the protonated electron deficient species 7 in a Minisci typepathway to provide $\mathbf{8}$. We presume that the intermediate $\mathbf{8}$ transfers another electron to the photoexcited carbon nitride VB to generate the desired product. Simultaneously, electrons accumulate in the $C B$ of carbon nitride, which are quenched by oxygen or reactive oxygen species to close the redox cycle. Under anaerobic condition, the photoexcited electrons can be 
transferred to Pt nanoparticles to produce $\mathrm{H}_{2}$.

In conclusion, we have achieved visible light mediated Minisci coupling of heteroarenes with ethers, alcohols and amides using carbon nitride as an inexpensive, non-toxic, easily synthesisable and scalable carbon-based photocatalyst. This photocatalytic method has improved performance compared to the state-of-the-art and displays a broad scope. Moreover, aerobic $\mathrm{O}_{2}$, an environmentally benign and freely available oxidant, is employed in this process instead of an external chemical oxidant under ambient condition. The photocatalyst is recyclable several times without any loss of activity. Furthermore, the process can be coupled with $\mathrm{H}_{2}$ production and thus making efficient use of the full redox cycle to generate two value-added products.

Scheme 1. Proposed mechanism of photocatalytic Minisci reaction.

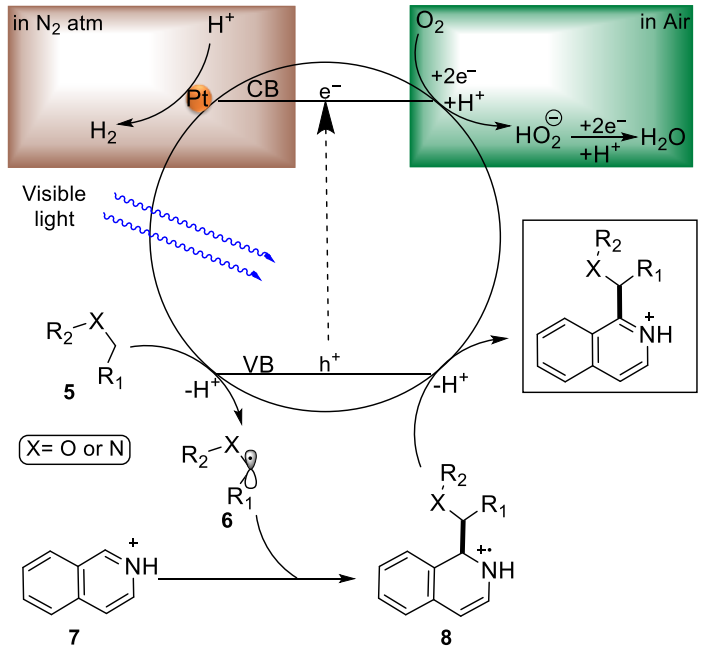

We would like to thank Dr. Fabio Lima, Prof. Steven Ley, Dr. Souvik Roy, Dr. Julien Warnan and Dr. Hatice Kasap for helpful discussions. The financial support from the Cambridge Trust and Science and Engineering Research Board, India, the Christian Doppler Research Association, Austrian Federal Ministry for Digital and Economic Affairs, National Foundation for Research, Technology and Development, and OMV is greatly appreciated.

\section{Conflicts of interest}

There are no conflicts to declare.

\section{Notes and References}

1 K. E. Dalle, J. Warnan, J. J. Leung, B. Reuillard, I. S. Karmel and E. Reisner, Chem. Rev., 2019, 119, 2752-2875.

2 X. Li, J. Yu, M. Jaroniec and X. Chen, Chem. Rev., 2019, 119, 3962-4179.

3 M. A. Ischay, M. E. Anzovino, J. Du and T. P. Yoon, J. Am. Chem. Soc., 2008 130, 12886-12887.

4 D. A. Nicewicz and D. W. C. MacMillan, Science, 2008, 322, 77-80.

5 J. M. R. Narayanam, J. W. Tucker and C. R. J. Stephenson, J. Am. Chem. Soc., 2009, 131, 8756-8757.

6 C. Stephenson, T. Yoon and D. W. C. MacMillan, Visible Light Photocatalysis in Organic Chemistry, Wiley-VCH Verlag GmbH \& Co. KGaA, Weinheim, Germany, 2018.

7 N. A. Romero and D. A. Nicewicz, Chem. Rev., 2016, 116, 10075-10166.
8 D. P. Hari and B. König, Chem. Commun., 2014, 50, 6688-6699.

9 X. Wang, K. Maeda, A. Thomas, K. Takanabe, G. Xin, J. M. Carlsson, K. Domen and M. Antonietti, Nat. Mater., 2009, 8, 76-80.

10 Y. Dai, C. Li, Y. Shen, T. Lim, J. Xu, Y. Li, H. Niemantsverdriet, F. Besenbacher, N. Lock and R. Su, Nat. Commun., 2018, 9, 60.

11 A. Savateev, I. Ghosh, B. König and M. Antonietti, Angew. Chem Int. Ed., 2018, 57, 15936-15947

12 F. Su, S. C. Mathew, G. Lipner, X. Fu, M. Antonietti, S. Blechert and X Wang, J. Am. Chem. Soc., 2010, 132, 16299-16301.

13 F. Su, S. C. Mathew, L. Möhlmann, M. Antonietti, X. Wang and S. Blechert, Angew. Chem. Int. Ed., 2011, 50, 657-660.

14 H. Kasap, C. A. Caputo, B. C. M. Martindale, R. Godin, V. W. Lau, B. V Lotsch, J. R. Durrant and E. Reisner, J. Am. Chem. Soc., 2016, 138, 91839192.

15 H. Kasap, D. S. Achilleos, A. Huang and E. Reisner, J. Am. Chem. Soc., 2018, 140, 11604-11607.

16 V. W. Lau, I. Moudrakovski, T. Botari, S. Weinberger, M. B. Mesch, V. Duppel, J. Senker, V. Blum and B. V. Lotsch, Nat. Commun., 2016, 7, 12165.

17 V. W. Lau, D. Klose, H. Kasap, F. Podjaski, M.-C. Pignié, E. Reisner, G. Jeschke and B. V. Lotsch, Angew. Chem. Int. Ed., 2017, 56, 510-514.

18 A. U. Meyer, V. W. Lau, B. König and B. V. Lotsch, Eur. J. Org. Chem, 2017, 2179-2185.

19 A.B. Charette, Handbook of Reagents for Organic Synthesis: Reagents for Heteroarene Functionalization, Wiley, 2015.

20 R. S. J. Proctor and R. J. Phipps, Angew. Chem. Int. Ed., 2019, DOI:10.1002/anie.201900977.

21 W. Buratti, G. P. Gardini, F. Minisci, F. Bertini, R. Galli and M. Perchinunno, Tetrahedron, 1971, 27, 3655-3668.

22 F. Minisci, A. Citterio, E. Vismara and C. Giordano, Tetrahedron, 1985, 41, 4157-4170.

23 J. K. Matsui, D. N. Primer and G. A. Molander, Chem. Sci., 2017, 8, 3512 3522.

24 C. A. Correia, L. Yang and C. J. Li, Org. Lett., 2011, 13, 4581-4583.

25 S. Liu, A. Liu, Y. Zhang and W. Wang, Chem. Sci., 2017, 8, 4044-4050.

26 Q.-Q. Wang, K. Xu, Y.-Y. Jiang, Y.-G. Liu, B.-G. Sun and C.-C. Zeng, Org Lett., 2017, 19, 5517-5520.

27 R. A. Garza-sanchez, A. Tlahuext-aca, G. Tavakoli and F. Glorius, ACS Catal., 2017, 7, 4057-4061.

28 J. Jin and D. W. C. MacMillan, Angew. Chem. Int. Ed., 2015, 54, 15651569.

29 J. Jin and D. W. C. MacMillan, Nature, 2015, 525, 87-90.

30 C. Bosset, H. Beucher, G. Bretel, E. Pasquier, L. Queguiner, C. Henry, A Vos, J. P. Edwards, L. Meerpoel and D. Berthelot, Org. Lett., 2018, 20 6003-6006.

31 R. S. J. Proctor, H. J. Davis and R. J. Phipps, Science, 2018, 360, 419-422.

32 C. Y. Huang, J. Li, W. Liu and C. J. Li, Chem. Sci., 2019, 10, 5018-5024.

33 E. Reisner, Angew. Chem. Int. Ed., 2019, 58, 3656-3657.

34 B. C. M. Martindale, G. A. M. Hutton, C. A. Caputo and E. Reisner, J. Am Chem. Soc., 2015, 137, 6018-6025.

35 B. C. M. Martindale, G. A. M. Hutton, C. A. Caputo, S. Prantl, R. Godin, J. R. Durrant and E. Reisner, Angew. Chem. Int. Ed., 2017, 56, 6459-6463.

36 T. Fuchigami, M. Atobe and S. Inagi, Fundamentals and applications of organic electrochemistry: synthesis, materials, devices, John Wiley \& Sons, Ltd, 2015

37 G. A. M. Hutton, B. C. M. Martindale and E. Reisner, Chem. Soc. Rev., 2017, 46, 6111-6123.

38 F. Ossola, G. Pistoia, R. Seeber and P. Ugo, Electrochim. Acta, 1988, 33, 47-50.

39 J. L. Jeffrey, J. A. Terrett and D. W. C. MacMillan, Science, 2015, 349, 1532-1536.

40 T. McCallum, S. P. Pitre, M. Morin, J. C. Scaiano and L. Barriault, Chem Sci., 2017, 8, 7412-7418.

41 L. Niu, J. Liu, X.-A. Liang, S. Wang and A. Lei, Nat. Commun., 2019, 10, 467. 


\section{TOC artwork}

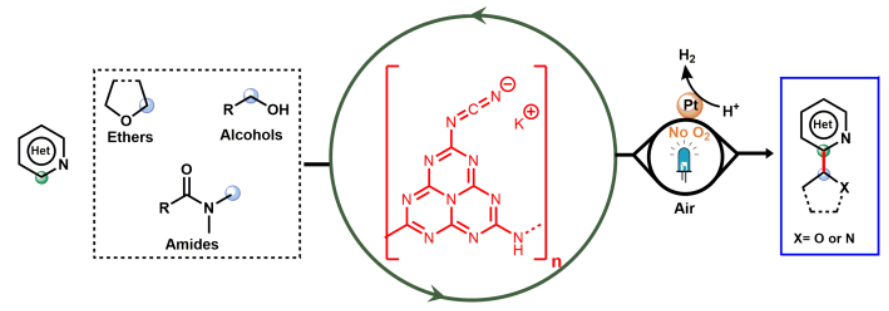

